\title{
Endogenous ROS levels in C. elegans under exogenous stress support revision of oxidative stress theory of life-history tradeoffs
}

\author{
Samson W Smith ${ }^{1,2}$, Leigh C Latta IV ${ }^{3}$, Dee R Denver ${ }^{4}$ and Suzanne Estes ${ }^{1 *}$
}

\begin{abstract}
Background: The oxidative stress theory of life-history tradeoffs states that oxidative stress caused by damaging free radicals directly underpins tradeoffs between reproduction and longevity by altering the allocation of energetic resources between these tasks. We test this theory by characterizing the effects of exogenous oxidative insult and its interaction with thermal stress and diet quality on a suite of life-history traits and correlations in Caenorhabditis elegans nematodes. We also quantify demographic aging rates and endogenous reactive oxygen species (ROS) levels in live animals.

Results: Our findings indicate a tradeoff between investment in reproduction and antioxidant defense (somatic maintenance) consistent with theoretical predictions, but correlations between standard life-history traits yield little evidence that oxidative stress generates strict tradeoffs. Increasing oxidative insult, however, shows a strong tendency to uncouple positive phenotypic correlations and, in particular, to reduce the correlation between reproduction and lifespan. We also found that mild oxidative insult results in lower levels of endogenous ROS accompanied by hormetic changes in lifespan, demographic aging, and reproduction that disappear in combined-stress treatments-consistent with the oxidative stress theory of aging.
\end{abstract}

Conclusions: Our findings demonstrate that oxidative stress is a direct contributor to life-history trait variation and that traditional tradeoffs are not necessary to invoke oxidative stress as a mediator of relationships between life-history traits, supporting previous calls for revisions to theory.

Keywords: Aging, Fitness, Free radicals, Lifespan, Resource allocation

\section{Background}

Evolutionary life-history theory predicts that tradeoffs; i.e., negative correlations, between fitness-related phenotypes will occur as a result of physiological constraints and/or resource limitations [1-4]. Tradeoffs have been widely hypothesized to result from organisms having limited energy stores, the allocation of which will evolve to favor either reproduction or somatic maintenance and longevity [5-7]; see [1] for a review. Commonly demonstrated tradeoffs include 'costs of reproduction' wherein fecundity is negatively correlated to other traits such as lifespan and susceptibility to oxidative and other forms of stress $[2,8,9]$. Reports of positive or variable

\footnotetext{
* Correspondence: estess@pdx.edu

'Department of Biology, Portland State University, 97201 Portland, OR, USA Full list of author information is available at the end of the article
}

correlations between traits where classic theory would predict strong negative correlations $[10,11]$ have been attributed to the expanded resource budgets typical under laboratory conditions; experimentally limiting resources, it has been suggested, might therefore illuminate true tradeoffs by forcing resource reallocation [11,12]. Accordingly, negative trait correlations have sometimes been revealed by manipulating food availability and by modulating various stressors $[2,9,13,14]$; however, others have highlighted weaknesses with the design of such experiments (e.g., introduction of confounding factors such as diet restriction) and argued that resource allocation models may be inadequate to explain observed relationships between life-history traits [15].

Despite such criticisms, a popular idea among evolutionary ecologists has been that oxidative stress caused 
by damaging free radicals shapes life histories [16] and, through its effects on resource allocation, life-history tradeoffs such as that between reproduction and somatic maintenance-a subject of much recent debate [17-21]. Free radicals including reactive oxygen species (ROS) are natural byproducts of aerobic energy metabolism occurring via oxidative phosphorylation at mitochondrial electron transport chains (ETC.) [22]. Organisms have evolved diverse, sophisticated mechanisms to neutralize ROS and protect against or repair oxidative damage; however, mitochondrial ETC. dysfunction and various environmental stressors (e.g., UV and thermal stress) can cause elevated levels of ROS and associated cellular damage [23-25] known to accumulate with age [25-30]. Oxidative stress results when an imbalance between ROS production and detoxification/repair allows such damage to accrue. Despite their capacity for harm, ROS and other free radicals have vital roles in immune system function, calcium homeostasis, and cell signaling $[25,31,32]$. Organisms must therefore satisfy the requirements of their dependence on mitochondrial ATP production while regulating levels of detrimental ROS byproducts; they must also maintain the beneficial roles associated with ROS while minimizing oxidative damage. In a model where ROS mediate life-history tradeoffs, elevated oxidative damage would shift the allocation of available resources among these tasks. The basic idea is that, when confronted with oxidative stress, organisms can devote energy to reproduction, leaving fewer resources for somatic maintenance (e.g., antioxidant protection or repair of oxidatively damaged biomolecules) and resulting in lasting oxidative damage that directly causes aging and shortens lifespan $[3,16]$. Alternatively, resources can be invested into somatic maintenance, enhancing survival and longevity at the expense of investments in other energetically demanding functions like reproduction.

A few key criticisms of this idea have been highlighted [12,33-35]. First, despite the fact that oxidative damage accumulates with age and has been linked to etiology of degenerative human diseases, whether it directly causes senescence and shortens lifespan is still unclear. For example, studies that have modified antioxidant levels generally support the idea that both exogenously and endogenously-acquired antioxidants protect against acute oxidative stress [35,36], but fail to find consistent effects of these treatments on lifespan in laboratory environments [35,37-41]. Second, maintaining antioxidant protective mechanisms may not be especially energetically costly, although there are exceptions (e.g., mitochondrial uncoupling proteins and glutathione). If organisms do not have to "choose" between devoting resources to reproduction or protective defenses, experiments aiming to force tradeoffs between these expenditures by limiting resources are misguided.
While such findings cast doubt on oxidative stress theories of aging and life-history tradeoffs, most researchers still agree that oxidative stress probably underlies some aspects of aging and may help to determine the span of healthy aging-even if it fails to determine maximal lifespan and related tradeoffs. Consequently, there have been calls for research that shifts the focus from maximal lifespan to other measures of aging [42] while making some account of the interacting factors that determine levels of ROS and associated oxidative damage. Furthermore, it has recently been suggested that experimentally manipulating reproductive effort is the only way to properly test a key prediction of the oxidative stress theory of tradeoffs: that increased reproductive effort is associated with (or directly causes) increased oxidative damage; e.g., [21]. The central argument is that measuring oxidative damage in relation to unmanipulated reproductive effort (especially when resources are not limiting) is unlikely to reveal a substantial cost to reproduction since individuals can alter their reproductive output to balance current with future reproductive efforts. This balancing act is likely influenced by organismal condition such that high-condition individuals better able to acquire resources can both reproduce at high rates and live long, resulting in positive life-history correlations. Unfortunately, experimentally manipulating reproductive output is difficult or impossible in many study organisms (especially in those without parental care) without also introducing undesired side effects. In such organisms, the best available approach may be to manipulate oxidative stress in ways that impose physiological constraints or resource limitations (e.g., access to exogenous antioxidants) while controlling for organismal condition, variation in resource acquisition, and other confounding factors such as food availability. While perhaps less likely to reveal strict tradeoffs than direct manipulations of reproductive effort, such an approach may provide insight into the frequency and extent to which tradeoffs are expected to arise in organisms experiencing endogenous or exogenous oxidative insult.

We investigated the role of ROS in mediating lifehistory variation and tradeoffs in a single genotype of $C$. elegans, an organism for which tradeoffs appear to be largely absent under standard laboratory conditions [43] but see [44]. We chronically exposed nematodes to different levels of paraquat-an herbicide that generates superoxide anions in vivo $[45,46]$ and measured a suite of life-history traits and rates of mortality and demographic aging. Additionally, we measured ROS levels using a fluorogenic dye-based method that allowed us to approximate the treatment-specific levels of oxidative stress experienced by nematodes [47]. We performed additional assays pairing oxidative challenge with mild thermal or dietary stress, treatments expected to further elevate oxidative stress, to test whether life-history tradeoffs 
would be further exposed or exhibit context-dependent expression.

\section{Methods}

\section{Nematode strains and culture conditions}

Bristol N2 Caenorhabditis elegans, obtained from the Caenorhabditis Genetics Center (University of Minnesota, St. Paul, MN), were used for all experiments. Nematode stocks were stored at $-80^{\circ} \mathrm{C}$ [48] and thawed as needed for all assays. Worms were age-synchronized by standard bleach treatment prior to each assay and grown at either $20^{\circ} \mathrm{C}$ or $25^{\circ} \mathrm{C}$ on $60 \mathrm{~mm}$ Petri plates containing NGM media with $20 \mu \mathrm{g} / \mathrm{mL}$ streptomycin and OP50-1 Escherichia coli as a food source. C. elegans were acclimated to their respective temperature and diet regimes (below) for two generations prior to each experiment.

\section{Paraquat $(\mathrm{PQ})$ treatments}

Paraquat (PQ), also methyl viologen dichloride, generates superoxide anions in vivo [49-52] and is commonly used to experimentally generate exogenous oxidative stress [49-51]. NGM plates were supplemented with: 0, 3.23, 32.3 or $64.6 \mu \mathrm{M}$ PQ in final concentration, hereafter referred to as control, low, medium and high PQ treatment levels, respectively. Nematodes were first exposed to their respective treatments at the egg stage on unseeded plates following age-synchronization. PQ was diluted in sterile M9, which served as the control treatment, and stock solutions were stored at $4^{\circ} \mathrm{C}$ and made fresh prior to each assay. Two hours prior to plate use, $0.5 \mathrm{~mL}$ of the stock PQ solution was added to the surface of each containing $15 \mathrm{~mL}$ medium and a lawn of OP50-1 E. coli. PQ treatment concentrations were decided upon in preliminary life history assays; PQ levels that dramatically reduced or prevented nematode development to maturity under otherwise standard conditions were avoided.

\section{Temperature treatments}

Phenotypic assays were conducted at either $20^{\circ} \mathrm{C}$ or $25^{\circ} \mathrm{C}$. $20^{\circ} \mathrm{C}$ is considered the thermal optimum for C. elegans based on intrinsic growth rate measurements (e.g., [53]), although this species has broad thermal tolerance and exhibits weak temperature preference [54]. $25^{\circ} \mathrm{C}$ assays were used to evaluate the effect of mild thermal stress on C. elegans' response to oxidative insult. This temperature results in faster generation time and reduced lifespan through processes including a general increase in enzyme activities [55], and reduced reproductive output of $C$. elegans compared to $20^{\circ} \mathrm{C}$ [10]. The lower reproductive output observed at $25^{\circ} \mathrm{C}$ is primarily due to a reduced number of functional sperm rather than to differences in oocyte production or egg hatching rates [56]. C. elegans is an androdioecious (hermaphrodite/rare male) species in which reproduction under standard laboratory conditions is predominantly sperm limited [56,57]. Hermaphrodite spermatogenesis is completed during the final larval stage (L4), while oogenesis begins during young adulthood [58,59]; temperature-induced changes in developmental timing can thus alter the time available for production of one or both types of gametes and form the basis of a tradeoff between generation time and lifetime fecundity [60].

\section{Diet quality treatments}

C. elegans were fed a diet of either live or UV-killed OP50-1 E. coli. C. elegans cultured on UV-killed E. coli experience lifespan extension (e.g., Figure ten in [61]) with no reported cost to reproductive output. This lifespan extension is unlikely to result from dietary restriction $[61,62]$ or the effects of laboratory adaptation [63]. Rather, worms raised on UV-killed bacteria avoid harmful bacterial proliferation within the gut [61]. However, worms will also lose any potential benefit of feeding on live bacteria; e.g., dietary determinants of lifespan such as coenzyme Q [64]. Thus, despite its benefits for longevity, an UV-killed E. coli diet could be considered stressful to C. elegans in this regard. Plates for the life history assay using UV-killed bacteria were incubated at $37^{\circ} \mathrm{C}$ for 4 hours to grow a light bacterial lawn and then irradiated for 2 hours at $76 \mathrm{~mJ} / \mathrm{cm}^{2}$ in a Logic Class II Type A2 biosafety cabinet (Labconco, Kansas City, MO) containing bulbs producing 253-nm radiation. Plates were stored overnight at room temperature and those with any sign of still-growing OP50-1 colonies were discarded.

\section{Relative reactive oxygen species (ROS) levels}

We measured steady-state ROS in live animals using a method that indicates net oxidant levels and thus reflects the rates of both ROS generation and scavenging by antioxidant systems $[65,66]$. We previously found that ROS levels measurement in this way were highly positively correlated (Spearman's $\rho=0.943, p=0.017$ ) with a survey of oxidative DNA damage (frequency of 8-oxo-dG lesions) conducted on experimentally evolved lines of N2 $C$. elegans [47], suggesting that this method can approximate the level of oxidative stress experienced by an organism. ROS levels were measured for live PQ-treated nematodes raised on live $E$. coli at either $20^{\circ} \mathrm{C}$ (n $\sim 80$ per PQ treatment; $\mathrm{N}=316$ ) or $25^{\circ} \mathrm{C}$ ( $\mathrm{n} \sim 80$ per PQ treatment; $\mathrm{N}=319$ ). (The $\mathrm{PQ}+\mathrm{UV}$-killed $E$. coli treatments yielded too few and developmentally asynchronous worms for meaningful ROS measurements). Briefly, ROS levels were assessed using confocal imaging of the pharyngeal bulb region of live nematodes labeled with MitoSOX Red (Invitrogen, Carlsbad, CA), a mitochondria-targeted dye that fluoresces when in contact with total mitochondrial oxidants [67]. MitoSOX Red working solutions were prepared as part of the respective PQ treatments and made 
from a fresh $5 \mathrm{M}$ MitoSOX Red stock in DMSO. A high resolution wide field Core DV system (Applied Precision) equipped with an Olympus IX71 inverted microscope mounted with a Nikon Coolsnap ES2 HQ camera (Advanced Light Microscopy Core Facility, Oregon Health and Science University, Portland, OR) was used to capture images at $60 \mathrm{X}$ magnification with a 0.15 second exposure time and $5 \mu \mathrm{m}$ z-stack widths. ROS levels were acquired by manually enclosing the terminal pharyngeal bulb within each deconvolution-optimized image and obtaining the maximum intensity of the area using ImageJ software (NIH). We applied this procedure to 40 labeled and 10 unlabeled (treatment-specific control) animals for each PQ treatment and calculated final ROS levels as the difference between average maximum pixel intensities (across z-stacks for each individual) for labeled and control worms.

We assessed ROS levels at two carefully chosen time points for nematodes within each treatment group: 34 and 68 hours after L1 stage arrest at $20^{\circ} \mathrm{C} ; 24$ and 48 hours after $\mathrm{L} 1$ stage arrest at $25^{\circ} \mathrm{C}$. This timing was chosen based on extensive preliminary study and observed times to reproductive maturation (below) and allowed us to image animals at similar developmental ages; i.e., during larval and reproductively mature stages in each treatment. However, because developmental rates varied among PQ treatment groups, measures of PQ-treated C. elegans reflect ROS levels at identical chronological (not developmental) ages. Assaying ROS at exactly identical developmental time points for these worms was not possible since this would necessitate slower-developing worms being exposed to PQ and the florescent label longer than faster-developing worms.

\section{Life history assays}

To determine the independent and combined effects of the $\mathrm{PQ}$, temperature, and diet quality treatments on fitness-related traits, we conducted three separate lifehistory assays: two using a live $E$. coli food source - one at $20^{\circ} \mathrm{C}(\mathrm{n}=200)$ and one at $25^{\circ} \mathrm{C}(\mathrm{n}=240)-$ and a third assay using UV-killed $E$. coli at $20^{\circ} \mathrm{C}(\mathrm{n}=200)$. For all assays, single L1 stage larvae were transferred from age synchronous populations to individual NGM plates and then transferred daily to fresh plates for at least five days following the first reproductive event to separate them from their progeny. 'Early reproduction' was calculated as the number of surviving offspring produced during the first two days of reproduction in the control treatment; 'late reproduction' was calculated as the total number of offspring for any remaining days of reproduction (c.f., [68]). Total lifespan was calculated as number of days from L1 arrest until death; survival rates were calculated from the same dataset. Worms were checked daily for survival and data were censored for nematodes that desiccated after crawling onto the side of a plate, which became more frequent in increasingly stressful conditions (Results).

Data from the life history assays were used to calculate relative fitness $(\omega)$ and age-specific mortality patterns for worms within each assay. Relative fitness was computed for each individual following ([69], eq. 2) as: $\omega=\sum e^{-r x} l$ (x) $m(x)$, where $l(x)$ is the number of worms surviving to day $x$ and $m(x)$ is the fecundity at day $x$, and where $r$ is the mean intrinsic population growth rate of the control treatment worms from the appropriate assay. The latter was calculated by solving Euler's equation, $\omega e^{-r x} l(x) m$ $(x)=1$, for $r$.

For comparative purposes and to further assess whether our experimental treatments differentially affected mortality dynamics, we estimated demographic aging parameters for each PQ treatment and assay. Initial mortality (IMR or "frailty") and rate of aging (ROA) were estimated by fitting survivorship data to a Gompertz mortality model, which assumes an exponential increase in mortality rate with age [70,71]. We also report mortality rate doubling time $(\mathrm{MRDT})$ and the median age at which survival $=50 \%$ for each assay and treatment as described in [42].

\section{Maturation rate}

Maturation rate; i.e., time to first reproductive event, was assessed for control and PQ-treated worms at each temperature ( $\mathrm{n} \sim 40$ per PQ treatment in each assay; $\mathrm{N}=298$ ). Beginning either 50 hours $\left(20^{\circ} \mathrm{C}\right)$ or 30 hours $\left(25^{\circ} \mathrm{C}\right)$ after L1 stage, nematodes were surveyed hourly for eggs using a dissecting microscope. Start times for these surveys were determined from preliminary experiments and each treatment cohort was removed from the incubator for scoring for identical lengths of time. Once the first eggs were identified, the parental hermaphrodite was sacrificed and the plate incubated overnight to confirm egg viability.

\section{Statistical analyses}

Only relative fitness from the $20^{\circ} \mathrm{C}$ live $E$. coli assay and late-life reproduction from the $20^{\circ} \mathrm{C}$ UV-killed $E$. coli assay conformed to normality (Shapiro-Wilk, $\alpha=0.05$ ). Attempts to normalize other datasets through transformation failed for all traits except ROS levels in the $25^{\circ} \mathrm{C}$ assay. Data for these traits were therefore analyzed both by non-parametric analysis (Wilcoxon/Kruskal-Wallis tests using rank sums) and one-way analyses of variance (ANOVA). When results of these tests agreed, parametric results are reported. Because the three assays could not feasibly be conducted simultaneously, any potential effects of experimental block would not be distinguished. Thus, no formal statistical comparisons are made between assays. We determined whether traits differed among particular pairs of $\mathrm{PQ}$ treatment levels within each temperature 
regime using the Tukey-Kramer HSD test or the nonparametric Wilcoxon method. The above analyses were performed in JMP 9.0.2 (SAS Statistical Inc., Cary, NC). Phenotypic variances, covariances, correlations and bootstrap estimates of their standard errors were generated using H2boot software [72].

\section{Results}

\section{In vivo ROS levels}

Because development was severely delayed in higher PQ-treatment groups with UV-killed E. coli (below), ROS experiments were restricted to the two live E. coli treatments. At $20^{\circ} \mathrm{C}$, larva exhibited fairly stable in vivo ROS levels with increasing $P Q$, but had significantly reduced ROS at the low PQ level (Tukey-Kramer HSD, $\alpha=0.05$; Figure $1 \mathrm{~A}$ closed symbols). By contrast, young adult worms showed overall higher ROS levels than larval worms, and a general trend of declining ROS with higher PQ treatment (Figure 1A open symbols). At $25^{\circ} \mathrm{C}$, ROS levels of larval worms declined in an approximately linear fashion from control to medium PQ level followed by a significant increase at the highest PQ level (Tukey-Kramer HSD, $\alpha=0.05$; Figure 1B closed symbols). Older worms exhibited a variable pattern of ROS level among control, low and medium PQ levels with a sharp decline at the highest PQ level (Figure 1B open symbols). The low ROS level observed for the latter group likely result from their unavoidably delayed development (see Methods). In particular, while control-, low- and medium-PQ-treated worms had reached the young adult stage at the time of these measurements, high-PQ-treated worms were still larvae. Finally, we note that a direct comparison between the $20^{\circ} \mathrm{C}$ and $25^{\circ} \mathrm{C}$ ROS experiments (Figure 1A versus $\mathrm{B}$ ) cannot be made owing to the confocal imaging system filter set and lenses having been changed between studies. However, data within each assay as well as the general patterns of difference between assays can be confidently compared.

\section{Mortality during life-history assays}

Initial sample sizes in each of the four PQ treatments were $\mathrm{n}=50$ (in the $20^{\circ} \mathrm{C}$ assays with live and UV-killed E. coli) or $\mathrm{n}=60$ (in the $25^{\circ} \mathrm{C}$ assay with live E. coli). Final sample sizes for each assay and PQ treatment group are provided in Additional file 1: Tables S1-S3. Differences between these numbers reflects data censored for worms that died from desiccation after crawling up the sides of their Petri plates, which became increasingly common in the combined stress treatments and accounted for $54 \%$ of the deaths in the highest PQ treatment with UV-killed E. coli. Data from these individuals were not considered in any of the analyses that follow.

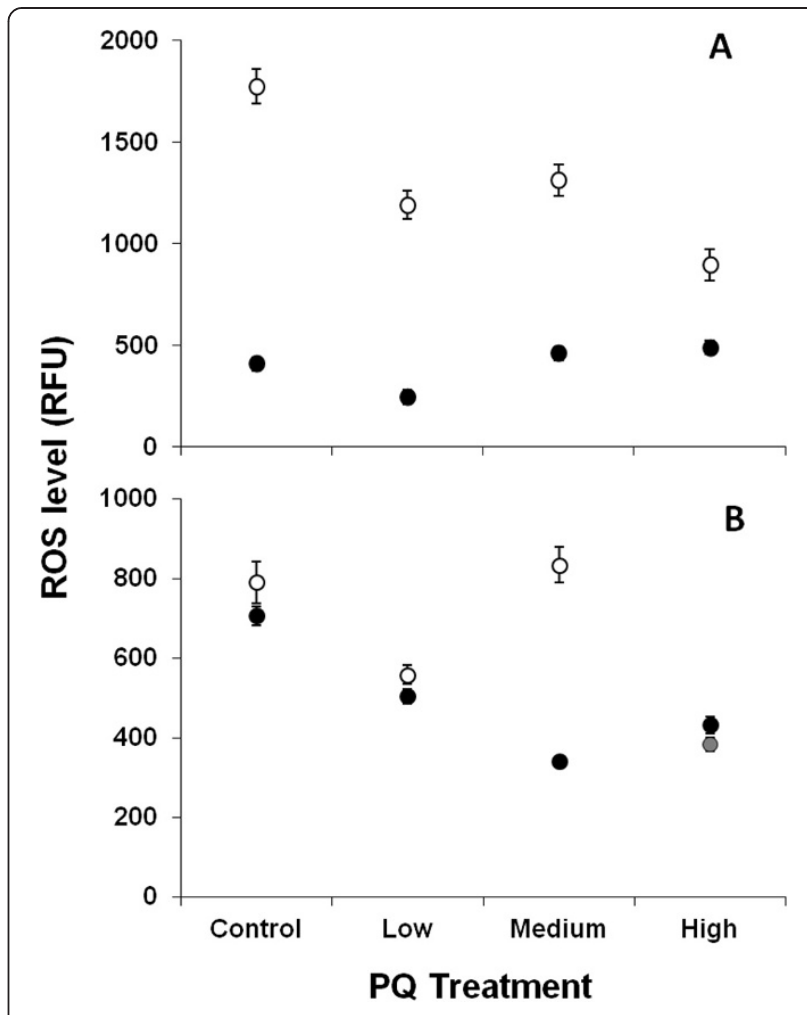

Figure 1 Relative steady-state ROS levels. ROS measured in relative fluorescence units of $\mathrm{PQ}$-treated nematodes fed live $E$. coli and raised at either $20^{\circ} \mathrm{C}$ (A) or $25^{\circ} \mathrm{C}$ (B). Filled symbols show ROS levels measured at earlier, developmentally similar (larval) time points ( $34 \mathrm{~h}$ at $20^{\circ} \mathrm{C}$ and $24 \mathrm{~h}$ at $25^{\circ} \mathrm{C}$ ); open symbols show later, mostly developmentally similar (reproductively mature) time points $\left(68 \mathrm{~h}\right.$ at $20^{\circ} \mathrm{C}$ and $48 \mathrm{~h}$ at $25^{\circ} \mathrm{C}$ ) for each assay. Note that the data point for older individuals in the High $\mathrm{PQ}$ treatment at $25^{\circ} \mathrm{C}$ (grey filled symbol) should be interpreted with caution due to the extremely delayed development of this group, and that the change in scale between the $y$-axes of (A) and (B) is not meaningful (see Results). Bars show one SEM. ANOVA revealed effects of $P Q$ level $\left(F_{3,316}=3.320, P=0.0202\right)$, age $\left(F_{1}=440.1, P<0.0001\right)$, and $P Q \times$ age $\left(F_{3}=21.51, P<0.0001\right)$ on ROS levels measured at $20^{\circ} \mathrm{C}$. The same was true for the $25^{\circ} \mathrm{C}$ assay with significant effects of $\mathrm{PQ}$ level $\left(F_{3,319}=63.39, P<0.0001\right)$, age $\left(F_{1}=36.71, P<0.0001\right)$ and $P Q x$ age $\left(F_{3}=67.33, p<0.0001\right)$ on ROS level.

\section{Reproductive output}

In the two assays using live E. coli, we observed the expected reduction in reproductive output of control worms raised at $25^{\circ} \mathrm{C}$ (Additional file 1: Table S2) compared to $20^{\circ} \mathrm{C}$ (Additional file 1: Table S1), and found that PQ significantly affected average total reproduction (Figure $2 \mathrm{~A}$ and $\mathrm{B}$ ).At $20^{\circ} \mathrm{C}$, animals experiencing any level of PQ exposure produced on average 12-24 more offspring than untreated controls (Additional file 1: Table S1). Similarly, in worms raised at $25^{\circ} \mathrm{C}$, reproductive output was higher for low-PQ (29 more offspring) and medium-PQ (23 more offspring) treatments compared to controls (Additional file 1: Table S2). Within the context of variable diet quality (but constant $20^{\circ} \mathrm{C}$ rearing temperature), total reproduction was affected by PQ 


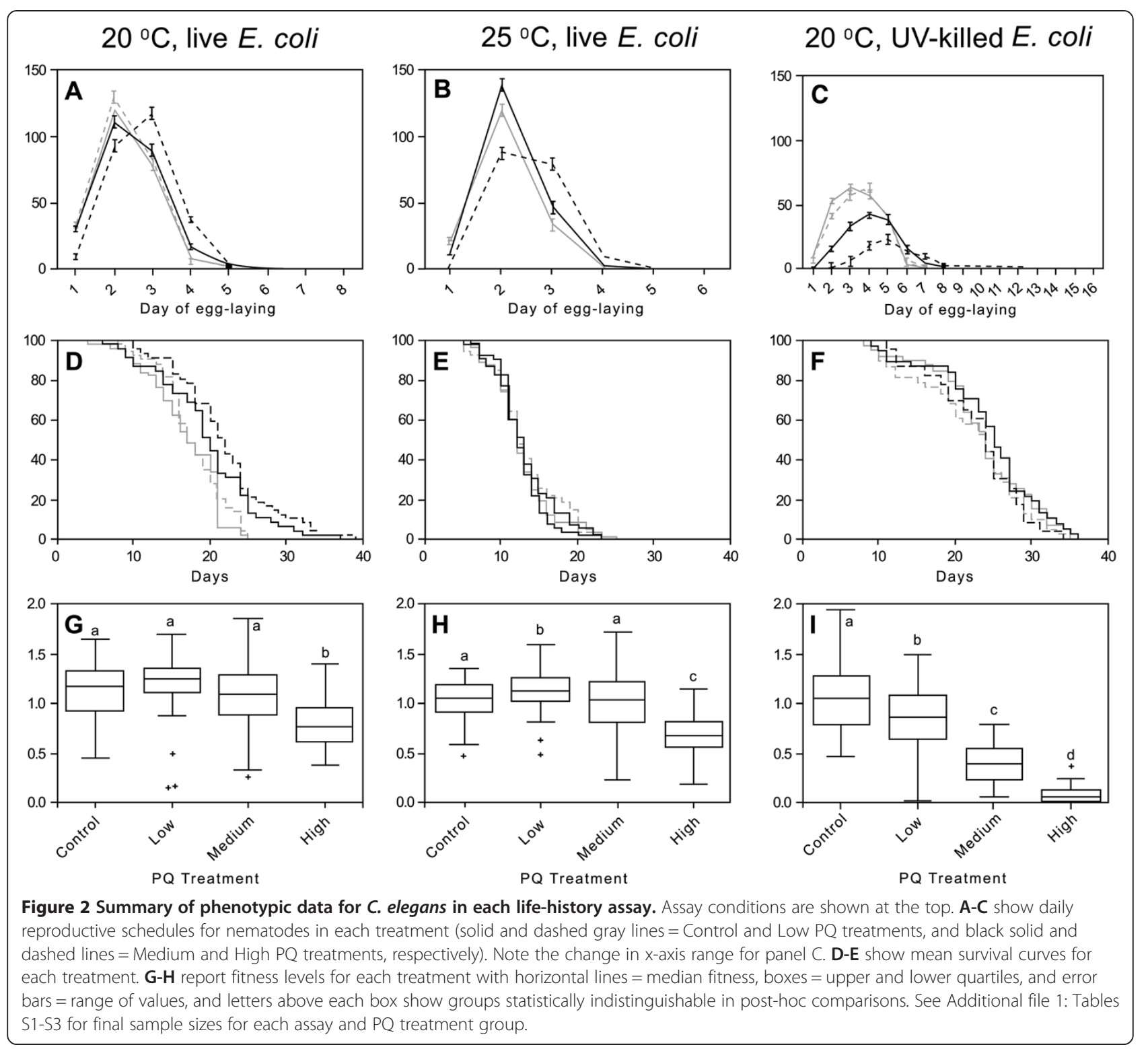

treatment (Figure 2A and C). Diet quality appeared to have little effect on this trait; for instance, there was no significant difference in mean reproduction of control worms fed live E. coli (Additional file 1: Table S1) and those fed UV-killed E. coli (Additional file 1: Table S3; $\mathrm{t}=1.40, \mathrm{P}=0.17$ ), with the caveat that these are from separate experiments. When faced with increasing PQ levels, however, nematodes fed live E. coli showed stable or slightly increased reproductive output (Additional file 1: Table S1) while reproduction in those fed UVkilled E. coli declined precipitously (Additional file 1: Table S3; Figure 2C). In summary, certain PQ treatment levels (oxidative insult) were associated with minor increases in reproduction, but only in worms fed live E. coli.

\section{Reproductive schedule}

In all assays, high PQ level resulted to varying degrees in a delay and extension of the reproductive period (Figure 2A-C). This effect was most pronounced in the assay using UV-killed E. coli (Figure 2C). High-PQ treated worms experienced the most severely delayed reproduction, but this delay did not affect mean total reproduction in worms reared on live E. coli (above, Additional file 1: Tables S1 and S2) as these animals increased their reproductive output on subsequent days (Figure 2A and B; Additional file 1: Tables S1 and S2). Conversely, higher-PQ treated worms cultured on UV-killed E. coli experienced a severe decline in total reproduction, which was not compensated by increased late-life reproduction (Figure 2C and Additional file 1: Table S3). 


\section{Maturation rate}

We measured time to reproductive maturity (Figure 3) to more precisely quantify differences in reproductive timing than in the standard life-history assays. Because time to reproduction was severely delayed in higher PQtreatment groups with UV-killed $E$. coli, we restricted these assays to the live $E$. coli treatments. The average time to first reproduction in control worms was substantially longer at $20^{\circ} \mathrm{C}$ as compared to $25^{\circ} \mathrm{C}$ (mean $\pm \mathrm{SE}$; $63.4 \pm 4.0$ versus $42.4 \pm 2.8$, respectively) and increasing PQ was associated with further delay in maturation rate. However, the pattern of differences among PQ treatments was strikingly similar at both temperatures; i.e., there were main effects of temperature and PQ on maturation rate, but no temperature-by-PQ interaction (Figure 3 legend).

\section{Longevity and demographic aging}

Compared to nematodes raised under standard laboratory conditions of $20^{\circ} \mathrm{C}$ with live E. coli (Figure 2D), those raised at $25^{\circ} \mathrm{C}$ showed the expected reductions in maximum lifespan and survival rate (Figure 2E) while those raised on UV-killed $E$. coli showed the expected increase in both traits (Figure $2 \mathrm{~F}$ ). In the $20^{\circ} \mathrm{C}$ assay with live E. coli, nematode survival curves differed significantly among PQ treatments (Log-Rank $\chi_{3}^{2}=27.40, P<0.0001$ )

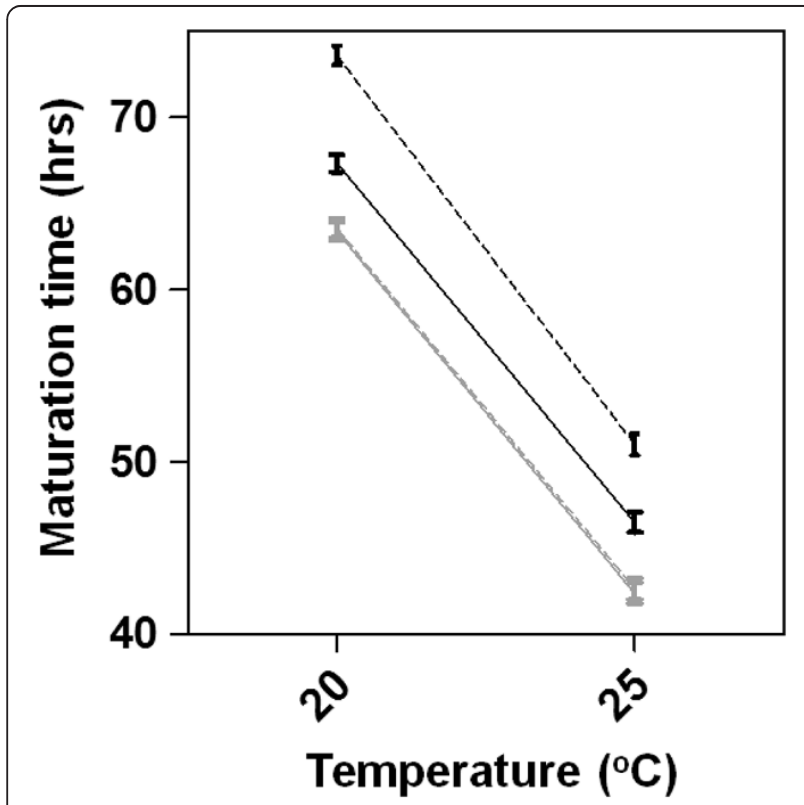

Figure 3 Maturation rates for $\mathrm{C}$. elegans raised at $20^{\circ} \mathrm{C}$ or $25^{\circ} \mathrm{C}$ on live $\boldsymbol{E}$. coli. Solid and dashed gray (overlapping) lines $=$ Control and Low PQ treatments, and black solid and dashed lines = Medium and High PQ treatments, respectively. Error bars represent one SEM; lines connect equivalent PQ treatments. ANOVA revealed highly significant effects of Temperature $\left(F_{1,298}=2695.9, P<0.0001\right)$ and $P Q$ level $\left(F_{3}=110.8, P<0.0001\right)$ but not their interaction $\left(F_{3}=0.950\right.$, $P=0.420$ ) on maturation rate measured as time in hours to first reproductive event. and were accompanied by a large ( 11 day) increase in maximum lifespan between the low and medium PQ treatments (Figure 2D). For the other two assays $\left(25^{\circ} \mathrm{C}\right.$ with live E. coli and $20^{\circ} \mathrm{C}$ with UV-killed E. coli), however, mean survival curves were nearly identical among PQ treatments (Figure 2E and F). The same patterns were observed for mean lifespan. Within the $20^{\circ} \mathrm{C}$ assay, mean lifespan showed a steady increase with PQ level (Additional file 1: Table S1); at $25^{\circ} \mathrm{C}$ lifespan showed the expected reduction compared the $20^{\circ} \mathrm{C}$ values, but was also remarkably stable with increasing PQ (Additional file 1: Table S2). Inspection of results from the two assays conducted at $20^{\circ} \mathrm{C}$ showed that, in contrast to the hormetic effect of PQ on the lifespan of worms fed live $E$. coli at $20^{\circ} \mathrm{C}$ (Additional file 1: Table S1), worms fed UV-killed bacteria had relatively long lifespans that were stable across PQ treatments (Additional file 1: Table S3). We note that worms that died from bagging (premature hatching of eggs within the parent) were included in the lifespan analyses as is standard in studies of nematode longevity, but these instances did not vary significantly among treatments $\left(12 \%, 11 \%\right.$, and $14 \%$ for $20^{\circ} \mathrm{C}$ and $25^{\circ} \mathrm{C}+$ live E. coli, and $20^{\circ} \mathrm{C}+\mathrm{UV}$-killed E. coli, respectively) and did not differ from control (no PQ) levels under standard conditions ( $14 \%$ for $20^{\circ} \mathrm{C}+$ live E. coli).

Additional file 1: Table S7 shows demographic aging parameters for each PQ treatment and assay, the patterns of which closely matched those of mean lifespan for this study. The key insights from these results were that aging slows with higher-PQ treatment, but only under standard laboratory conditions $\left(20^{\circ} \mathrm{C}\right.$ with live E. coli). Log-rank tests identified significant differences between survival curves of all pairs of PQ treatment groups in this assay except between the control and low levels and the medium and high levels (data not shown). Both higher temperature and UV-killed E. coli conditions masked the effect of PQ on rates of aging: the apparent benefit of PQ for aging disappeared at $25^{\circ} \mathrm{C}$ where aging was fast and more uniform across PQ levels; conversely, aging was uniformly slow with UV-killed E. coli (Additional file 1: Table S7). The same effects are illustrated by comparisons of survival curves for the control populations from each assay, showing that higher temperature significantly increased the rate of aging [Log-rank test for controls from $25^{\circ} \mathrm{C}$ vs. $20^{\circ} \mathrm{C}$ assays with live E. coli; difference in relative risk $(\mathrm{SE})=0.833(0.042), \mathrm{P}=0.0003$ ], while an $\mathrm{UV}$ killed bacterial diet reduced the rate of aging [Log-rank test for controls from $20^{\circ} \mathrm{C}$ with live E. coli vs. $20^{\circ} \mathrm{C}$ with UV-killed $E$. coli assays; difference in relative risk $(\mathrm{SE})=0.787$ (0.027), $\mathrm{P}<0.0001]$.

\section{Relative fitness}

Analysis of assays conducted using live $E$. coli detected a strong effect of PQ level but suggested that temperature 
had little influence on this trait. Although worms cultured at $25^{\circ} \mathrm{C}$ had reduced total reproduction and lifespan (Additional file 1: Table S2) compared to those cultured at $20^{\circ} \mathrm{C}$ (Additional file 1: Table S1), their strong earlylife reproduction allowed them to achieve similar levels of relative fitness (compare Figure $2 \mathrm{H}$ and $\mathrm{G}$ ). For both the $20^{\circ} \mathrm{C}$ (Figure $2 \mathrm{G}$ ) and $25^{\circ} \mathrm{C}$ (Figure $2 \mathrm{H}$ ) assays, mean relative fitness was fairly stable with increasing PQ until the highest PQ dose where it declined significantly (Tukey-Kramer HSD, $\alpha=0.05$ ). The magnitude of this decline relative to controls was less extreme at $20^{\circ} \mathrm{C}$ (Additional file 1: Table S1) than at $25^{\circ} \mathrm{C}$ (Additional file 1: Table S2), but in both cases was driven by the delayed (but not reduced) reproduction observed at the highest PQ level (Figure 2A and B; Figure 2). Analysis of both $20^{\circ} \mathrm{C}$ assays also revealed an effect of $\mathrm{PQ}$, and were consistent with an interaction effect of PQ with diet quality on relative fitness. Worms cultured on live E. coli (Figure 2H; Additional file 1: Table S1) showed greater resilience to PQ than worms fed UV-killed $E$. coli (Figure 2I; Additional file 1: Table S3). The latter group's relative fitness declined steadily with increasing $\mathrm{PQ}$, reaching nearly zero at the highest PQ level; this decline resulted from a combination of delayed reproduction (Figure $2 \mathrm{C}$ ) and lower fecundity (Additional file 1: Table S3).

\section{Phenotypic variance and life-history trait correlations}

Additional file 1: Tables S4-6 show individual trait variances and covariances and correlations between pairs of traits for each assay. Within assays, trait variances had a weak tendency to expand with increasing oxidative stress; however, variances tended to contract slightly under thermal stress (compare Additional file 1: Table S5 to S4).

Visual inspection of Figures 2 and 3 indicates that, at a coarse level, environmental stress may have induced lifehistory tradeoffs. Compared to worms raised at $20^{\circ} \mathrm{C}$ under typical lab conditions (Figures 2A,D and 3), those raised at $25^{\circ} \mathrm{C}$ tended to have slightly increased Day 2 fecundity (Figure $2 \mathrm{~B}$ ), probably due to their accelerated development (Figure 3), but faster aging and reduced longevity (Figure 2E); those raised on UV-killed E. coli showed the opposite pattern of tradeoff-far fewer offspring produced early in life (Figure $2 \mathrm{C}$ ) but slower aging and increased longevity (Figure 2F). These patterns are subtly evident in the proper phenotypic covariances where, for example, relationships between total reproduction and lifespan measured for control worms were positive under normal lab conditions (Additional file 1: Table S4) but significantly lower (Fisher's z-tests, $\mathrm{P}<0.01$ ) and indistinguishable from zero at both $25^{\circ} \mathrm{C}$ (Additional file 1: Table S6) and in UV-killed E. coli conditions (Additional file 1: Table S6). We observed the same pattern within the $20^{\circ} \mathrm{C}$ live $E$. coli assay where the correlation between these two traits declined monotonically from +0.535 to -0.030 with increasing PQ level (Additional file 1: Table S4). Fisher's z-tests revealed significant differences between correlations measured in control and high PQ treatment $(\mathrm{z}=$ 3.007, $\mathrm{P}=0.003)$ and in low and high PQ treatment $(\mathrm{z}=$ 2.055; $\mathrm{P}=0.040)$. Overall, similar patterns held within both assays using live E. coli (Additional file 1: Tables S4-S5) where the correlation between reproductive traits became increasingly negative with PQ level-except for high-PQ treated worms at $25^{\circ} \mathrm{C}$ where early reproduction and lifespan were significantly positively associated (Additional file 1: Table S5). The correlation between early- and late-life reproduction also grew increasingly negative at higher PQ levels in the live-E. coli assays, but the correlations achieved statistical significance only at $25^{\circ} \mathrm{C}$ (Additional file 1: Table S5). Although the same patterns did not hold for worms fed UV-killed $E$. coli, a major caveat is that they displayed essentially no variance in early reproduction (Additional file 1: Table S6); correlations involving this trait must therefore be interpreted with caution. In this, the most stressful environment, the magnitude and sign of trait relationships varied across PQ levels with little discernible pattern (Additional file 1: Table S6) but were overall more positive in sign than those measured in the other two assays.

Some of the measured traits (e.g., early and total reproduction) are necessarily positively correlated due to overlapping measurement; the magnitude of these correlations can nonetheless reveal the contributions of early- and late-life reproduction to total reproduction and fitness. In particular, late-life reproduction became more strongly correlated (i.e., contributed more) to total reproduction at higher PQ levels in all assays; the opposite pattern was observed for early-life reproduction (Additional file 1: Tables S4-S6). This was especially true in the 20C assay with UV-killed $E$. coli where late-life reproduction also became significantly positively correlated with relative fitness in the medium and high PQ treatments (Additional file 1: Table S6).

\section{Discussion}

\section{Nematode life-histories under thermal stress and altered} diet quality

Comparing data from control (no PQ) treatments across the three experimental environments yields insight into potential main effects of temperature and diet quality on C. elegans life histories. Consistent with previous results (e.g., $[10,56,73])$, worms cultured at $25^{\circ} \mathrm{C}$ had reduced lifespan, survival rate, maturation time, and total fecundity compared to those cultured at the optimal $20^{\circ} \mathrm{C}$. This altered life history did not, however, reveal strict tradeoffs (Additional file 1: Tables S4 and S5). Rather, phenotypic correlations between lifespan and all measured reproductive traits were statistically indistinguishable from zero at 
$25^{\circ} \mathrm{C}$; however, these correlations were uniformly lower than those measured at $20^{\circ} \mathrm{C}$. For example, the positive correlation $\left(r_{P}=0.535\right)$ observed between total reproduction and lifespan at $20^{\circ} \mathrm{C}$ (Additional file 1: Table S4) vanished at $25^{\circ} \mathrm{C}\left(\mathrm{r}_{\mathrm{P}}=-0.081\right.$; Additional file 1: Table S5), indicating that physiological stress may break positive correlations between traits.

The severe, negative effect of the UV-killed bacterial diet on daily reproductive output of control worms (Figure 2, Additional file 1: Table S3) suggested this was the most stressful of our treatments, but the negative consequences for reproduction were offset by increased mean survival, extended lifespan, and slowed rates of demographic aging (Additional file 1: Table S7), which allowed worms to maintain high relative fitness. Thus, this treatment resulted in tradeoffs in the classical sense; i.e., negative phenotypic correlations between lifespan and both early fecundity and total fitness (Additional file 1: Table S6). This result differs from previous reports that an UV-killed bacterial diet extends $C$. elegans lifespan in a cost-free manner; e.g., [74], but these studies reported only mean lifetime reproduction. We found that diet-stressed worms pay for their extended lifespan with delayed and reduced early reproduction. Even in the absence of $\mathrm{PQ}$, animals in this treatment likely experienced heightened oxidative stress (see Methods). A complication with using these results to directly test the oxidative stress theory of lifehistory tradeoffs, however, is that the life-history costs and benefits of this diet treatment may have different underlying causes: the life-extending effects of an UV-killed (or growth-arrested) bacterial diet are believed to arise from worms avoiding the harmful effects of bacterial proliferation in the gastrointestinal tract [61], which may or may not reduce levels of oxidative damage, while the costs of this diet for reproduction are likely to result from lost nutrients and/or reduced antioxidant defense [64]. Thus, the above findings cannot tell us whether oxidative stress is at the heart of observed lifehistory trait relationships, but lead to the preliminary conclusion that environmental stress tends to reduce the strength of the positive correlations between lifespan and reproduction measured in benign conditions-even it fails to reveal hidden tradeoffs.

\section{Treatment-specific oxidative stress levels}

We found that C. elegans responded to increasing PQ in both age- and temperature-dependent ways such that $25^{\circ} \mathrm{C}$ appeared more stressful than $20^{\circ} \mathrm{C}$ and younger worms were less oxidatively stressed than older worms (Figure 1). During larval stages, nematodes were capable of modulating their endogenous ROS levels under all PQ and temperature treatments, but worms of all stages likely experienced higher levels of oxidative stress in the higher temperature. As previously noted for the $25^{\circ} \mathrm{C}$ assay, older worms at the highest PQ level exhibited reduced oxidative stress (Figure $1 \mathrm{~B}$ grey symbol) as a likely consequence of this group's delayed development (Figure 3) meaning that it was comprised of larval worms at the time of measurement. Younger animals' superior responsiveness to oxidative insult is expected based on their antioxidant capacities: for example, steadystate mRNA levels for the ROS detoxification enzymes glutathione $\mathrm{S}$-transferase and superoxide dismutases were elevated in larval but not adult worms subjected to acute paraquat exposure [75]. Additionally, larval-stage C. elegans rely less strongly on metabolic pathways that generate endogenous ROS than do adult-stage worms [76]. Although our data are unavoidably confounded by variation in developmental rates (Figure 3; Methods) and any associated differences in antioxidant capacity or endogenous ROS generation among treatment groups, they shed light on how individuals in the life-history assays experienced the temperature and PQ treatments. Finally, that we failed to obtain sufficient numbers of agesynchronous animals from the UV-killed $E$. coli assay to measure ROS levels is further evidence that C. elegans experienced this as the most stressful treatment.

\section{Nematode life-histories in oxidative- and combined-stress treatments}

Chronic PQ exposure yielded slight hormetic effects on life-history traits in dose- and temperature-dependent ways, but only for worms fed live E. coli. PQ treatment increased total reproduction at both $20^{\circ} \mathrm{C}$ and $25^{\circ} \mathrm{C}$ (Figure 2, Additional file 1: Tables S1-S2). This effect was sustained across all PQ levels at the less stressful $20^{\circ} \mathrm{C}$ temperature, and was somewhat surprising since self-reproductive capacity in C. elegans is predominantly sperm-limited [60] and there was no opportunity for outcrossing in our life-history assays. This result may indicate that the negative effect of PQ treatment on developmental rate (Figure 3) allowed additional time for spermatogenesis to occur by extending the L4 stage (to which spermatogenesis is confined), resulting in higher total reproductive output. Also interesting was that the enhanced reproductive output of worms in the low-PQ treatments-where hormesis was most pronounced at both temperaturesappeared to come at no cost to other traits. Indeed, at $20^{\circ} \mathrm{C}, \mathrm{PQ}$ treatment also had hormetic effects on lifespan and rates of survival and demographic aging-unlike at $25^{\circ} \mathrm{C}$ where lifespan was short and aging was fast across all PQ levels. The favorable effects of medium- or high-PQ levels on nematode reproduction were, however, always accompanied by delayed development and reproductive timing. Although these worms compensated for lost early reproduction by increasing late-life reproductive output (c.f., [77]), the shifted reproductive schedules severely diminished relative fitness of worms in high-PQ treatments 
(Figure 2G and H). In light of the fact that ROS levels were significantly lower in low-PQ treated than in control worms at both temperatures (Figure 1), these findings suggest that mild oxidative insult is beneficial for $C$. elegans, resulting in lower endogenous ROS levels and cost-free hormetic effects on reproduction- and, in less stressful environments, aging-related traits. If these lower ROS levels reflect lower levels of oxidative damage as previously observed [47], mild oxidative insult may exert its hormetic effects through enhanced ROS scavenging (and reduced oxidative damage) resulting from the enhanced antioxidant gene expression known to occur in response to PQ [75]. Taken together, the above findings reaffirm that $25^{\circ} \mathrm{C}$ is the more stressful temperature and indicate that the threshold beyond which oxidative stress becomes costly is reached sooner for worms in combined stress (high temperature $+\mathrm{PQ}$ ) treatments.

In agreement with the above assertion, all hormetic effects of PQ disappeared when nematodes were raised with an UV-killed bacterial diet (Figure 2; Additional file 1: Tables S3 and S7), our most stressful experimental treatment. Reproductive traits were profoundly negatively affected by PQ treatment in this environment and, despite extended reproductive periods, worms failed to compensate for lost reproductive output later in life. In contrast to the step-wise negative effects of PQ on reproduction in this environment, the UV-killed diet improved survival, extended lifespan, and slowed rates of aging irrespective of PQ level. We speculate that animals in these treatments were pushed to the limits of their capacity to respond to oxidative damage and, in view of the marked decline in relative fitness with increasing PQ (Figure 2I), were aging in unhealthy ways despite being long lived. The more pronounced effects of oxidative insult observed in this environment may be due to reduced availability of exogenous antioxidants. In support of this idea, development of clk-1 mutants, which are unable to synthesize coenzyme $\mathrm{Q}\left(\mathrm{Q}_{9}\right)$, was arrested unless their diet was supplemented with an alternative quinone, $\mathrm{Q}_{8}$, from OP50 E. coli [78]. In addition to functioning as coenzymes within the mitochondrial ETC., quinones also serve as lipid-soluble free radical sinks. It is not known which other antioxidant molecules C. elegans obtains from its diet, but it is reasonable to suppose that only nematodes in the live E. coli experiments were acquiring an antioxidant buffer from their living bacterial food source whose own antioxidant systems are known to be upregulated in response to the concentrations of PQ used here [79-81] despite the known bacteriostatic effects of this compound [82]. We also acknowledge that these effects may be temperature dependent such that, for example, E. coli in the higher temperature treatment were better able to respond to paraquat; further study would be required to identify such interactions if they exist.
Although we were unable to quantify ROS for the long-lived worms in the UV-killed diet treatment, we noted that mean ROS levels in older worms (Figure 1A and $\mathrm{B}$, open symbols) declined alongside rates of aging (RoA; Additional file 1: Table S7) with increasing PQ at $20^{\circ} \mathrm{C}$, while both traits were uniformly higher at $25^{\circ} \mathrm{C}$. Maximal lifespan (Additional file 1: Tables S1 and S2) showed the opposite trend for both assays. Together with the observation that low PQ had other beneficial effects in the less stressful $20^{\circ} \mathrm{C}$ environment (above), these findings are consistent with the oxidative stress theory of aging [25], but do not provide a definitive test. In any case, the ROS data show that animals do not experience oxidative stress in direct proportion to exogenous treatment and highlight the benefits of measuring net oxidant levels in live tissue.

Finally, we found no overwhelming tendency for oxidative stress to generate negative correlations between traits measured on the same individuals. Increasing oxidative stress did, however, tend to decouple positive phenotypic correlations and, in particular, to break correlations between lifespan and reproduction-a pattern similar to the one described for control worms between environments. This trend was far more pronounced in the $20^{\circ} \mathrm{C}$ assay conducted with live E. coli (Additional file 1: Table S4) than in the other more stressful treatments where trait correlations became more variable (Additional file 1: Tables S5 and S6). Despite the absence of strict tradeoffs between standard life-history traits, we assert that delayed reproductive timing, which constitutes a major reproductive fitness disadvantage for nematodes (c.f., [83]) observed with increasing PQ levels (Figures 2 and 3) reflects a life-history tradeoff within the framework of somatic maintenance prioritization over reproduction. Because C. elegans' response to PQ exposure includes upregulating expression of energetically costly glutathione S-transferase [75], such a tradeoff could be mediated through increased allocation of resources to oxidative stress response at the expense of reproductive fitness. This interpretation makes sense in light of the shared molecular genetic mechanisms of survival and oxidative stress resistance in C. elegans such as the insulin and insulin-like growth factor signaling (IIS) and target of rapamycin (TOR) pathways. For instance, mild oxidative and other forms of environmental stress activate the DAF-16/FOXO transcription factor, a master regulator of longevity that is typically repressed by IIS. It is reasonable to believe that our PQ treatments cued the IIS, activating FOXO and signaling the downstream oxidative stress response system [84], leading ultimately to increased survival; e.g., [85]. This suspected tradeoff was more pronounced when nematodes were confronted with an UV-killed bacterial diet and loss of any exogenous antioxidants; here the hypothesized shift toward somatic maintenance (manifested 
as extreme developmental delay and extended lifespan) came at a major cost to reproductive output and relative fitness. Unfortunately, it was not possible to measure to ROS levels and life-history traits on the same individuals, which would have permitted us to calculate phenotypic correlations between these traits.

\section{Conclusions}

We observed complex and environmentally-dependent effects of oxidative insult on life-history traits that, viewed in light of physiological ROS levels, strongly imply that oxidative stress mediates the relationship between such traits. Criticisms of the oxidative stress theory for life-history tradeoffs are predicated on the absence of tradeoffs sensu stricto. Although our study could not avoid all previously criticized aspects of experimental design (e.g., quantifying oxidative stress based on tissuespecific measurement of a single class of oxidants), we nonetheless show that traditional tradeoffs are not necessary to invoke oxidative stress as a mediator of relationships between life-history traits, supporting the call for a revision of this theory $[20,86]$.

\section{Ethics}

This study does not fall under the approval process by Portland State University's Institutional Animal Care and Use Committee for the ethical use of research animals as it does not involve vertebrate species. This is in keeping with the requirements of the Animal Welfare Act and USDA regulations.

\section{Additional file}

Additional file 1: Table S1. C. elegans life-history variation among PQ levels at $20^{\circ} \mathrm{C}$ on live E. coli. Table S2. C. elegans life-history variation among PQ levels at $25^{\circ} \mathrm{C}$ on live E. coli. Table S3. C. elegans life-history variation among PQ levels at $20^{\circ} \mathrm{C}$ on UV-killed E. coli. Table S4. Phenotypic variances, covariances, and correlations for C. elegans at $20^{\circ} \mathrm{C}$ on live E. coli. Table S5. Phenotypic variances, covariances, and correlations for C. elegans at $25^{\circ} \mathrm{C}$ on live E. coli. Table S6. Phenotypic variances, covariances, and correlations for C. elegans at $20^{\circ} \mathrm{C}$ on UV-killed E. coli. Table S7. Treatment-specific estimates of demographic aging parameters.

\section{Competing interests}

The authors declare that they have no competing interests.

\section{Authors' contributions}

SWS and SE designed and executed the experiments. SWS, LCL, and SE analyzed the data. SWS, LCL, DRD, and SE wrote the manuscript. All authors read and approved the final manuscript.

\section{Acknowledgements}

We thank A. Snyder (OHSU Advanced Light Microscopy Core Facility) for technical advice on confocal imaging, J. Groody and S. Welker for lab assistance, I. M. Chelo, K. A. Hicks, J. Joyner-Matos for helpful discussion, P. C. Phillips for advice on data analysis, S. C. Harvey and two anonymous reviewers for helpful comments, and J. M. Sommers for observing that, "Life ain't nothin' but a funny, funny riddle". This work was funded by a Sigma Xi Grant-in-Aid of Research and an Equity Foundation Pride of the Rose Scholarship to SWS, and by NIH 5 R01 GM087628-02 to DRD and SE.

\section{Author details}

'Department of Biology, Portland State University, 97201 Portland, OR, USA. ${ }^{2}$ Current address: Department of Biology and Microbiology, South Dakota State University, 57007 Brookings, SD, USA. ${ }^{3}$ Biology Department, Reed College, 97202 Portland, OR, USA. ${ }^{4}$ Department of Zoology, Oregon State University, 97331 Corvallis, OR, USA.

Received: 22 April 2014 Accepted: 14 July 2014

Published: 24 July 2014

\section{References}

1. Stearns SC: Trade-offs in life-history evolution. Funct Ecol 1989, 3:259-268.

2. Zera AJ, Harshman LG: The physiology of life history trade-offs in animals. Annu Rev Ecol Syst 2001, 32:95-126.

3. Monaghan P, Metcalfe NB, Torres R: Oxidative stress as a mediator of life history trade-offs: mechanisms, measurements and interpretation. Ecol Lett 2009, 12:75-92.

4. Blount JD: Carotenoids and life-history evolution in animals. Arch Biochem Biophys 2004, 430:10-15.

5. Kirkwood TBL: Evolution of ageing. Nature 1977, 270:301-304.

6. Kirkwood TBL: Understanding the odd science of aging. Cell 2005, 120:437-447.

7. Kirkwood TB, Austad SN: Why do we age? Nature 2000, 408:233-238.

8. Alonso-Alvarez C, Bertrand S, Devevey G, Prost J, Faivre B, Sorci G: Increased susceptibility to oxidative stress as a proximate cost of reproduction. Ecol Lett 2004, 7:363-368.

9. Alonso-Alvarez C, Bertrand S, Devevey G, Prost J, Faivre B, Chastel O, Sorci G: An experimental manipulation of life-history trajectories and resistance to oxidative stress. Evolution (N Y) 2006, 60:1913-1924.

10. Klass MR: Aging in the nematode Caenorhabditis elegans: major biological and environmental factors influencing life span. Mech Ageing Dev 1977, 6:413-429.

11. Reznick D, Nunney L, Tessier A: Big houses, big cars, superfleas and the costs of reproduction. Trends Ecol Evol 2000, 15:421-425.

12. Van Noordwijk AJ, De Jong G: Acquisition and allocation of resources: their influence on variation in life history tactics. Am Nat 1986, 128:137-142.

13. Wang $Y$, Salmon AB, Harshman LG: A cost of reproduction: oxidative stress susceptibility is associated with increased egg production in Drosophila melanogaster. Exp Gerontol 2001, 36:1349-1359.

14. Chippindale AK, Chu TJF, Rose MR: Complex trade-offs and the evolution of starvation resistance in Drosophila melanogaster. Evolution (N Y) 1996, 50:753-766.

15. Speakman JR, Garratt M: Oxidative stress as a cost of reproduction: beyond the simplistic trade-off model. Bioessays 2014, 36:93-106.

16. Dowling DK, Simmons LW: Reactive oxygen species as universal constraints in life-history evolution. Proc R SOC B 2009, 276:1737-1745.

17. Metcalfe NB, Alonso-Alvarez C: Oxidative stress as a life-history constraint: the role of reactive oxygen species in shaping phenotypes from conception to death. Funct Ecol 2010, 24:984-996.

18. Harshman LG, Zera AJ: The cost of reproduction: the devil in the details. Trends Ecol Evol 2007, 22:80-86.

19. Selman C, Blount JD, Nussey DH, Speakman JR: Oxidative damage, ageing, and life-history evolution: where now? Trends Ecol Evol 2012, 27:570-577.

20. Gems D, Doonan R: Antioxidant defense and aging in C. elegans: Is the oxidative damage theory of aging wrong? Cell Cycle 2009, 8:1681-1687.

21. Metcalfe NB, Monaghan P: Does reproduction cause oxidative stress? An open question. Trends Ecol Evol 2013, 28:347-350.

22. Murphy MP: How mitochondria produce reactive oxygen species. Biochem J 2009, 417:1-13.

23. Cabiscol E, Tamarit J, Ros J: Oxidative stress in bacteria and protein damage by reactive oxygen species. Int Microbiol 2000, 3:3-8.

24. Wenk J, Brenneisen P, Meewes C, Wlaschek M, Peters T, Blaudschun R, Ma W, Kuhr L, Schneider L, Scharffetter-Kochanek K: UV-induced oxidative stress and photoaging. Curr Probl Dermatol 2001, 29:83-94.

25. Finkel T, Holbrook NJ: Oxidants, oxidative stress and the biology of ageing. Nature 2000, 408:239-247.

26. Beckman $\mathrm{KB}, \mathrm{Ames} \mathrm{BN}$ : The free radical theory of aging matures. Physiol Rev 1998, 78:547-581.

27. Klass M, Nguyen PN, Dechavigny A: Age-correlated changes in the DNA template in the nematode Caenorhabditis elegans. Mech Ageing Dev 1983, 22:253-263. 
28. Richter C, Park JW, Ames BN: Normal oxidative damage to mitochondrial and nuclear DNA is extensive. Proc Natl Acad Sci U S A 1988, 85:6465-6467.

29. Anson R, Bohr V: Mitochondria, oxidative DNA damage, and aging J Am Aging Assoc 2000, 23:199-218.

30. Hartman PS, Ishii N, Kayser EB, Morgan PG, Sedensky MM: Mitochondrial mutations differentially affect aging, mutability and anesthetic sensitivity in Caenorhabditis elegans. Mech Ageing Dev 2001, 122:1187-1201.

31. Tzou P, De Gregorio E, Lemaitre B: How Drosophila combats microbial infection: a model to study innate immunity and host-pathogen interactions. Curr Opin Microbiol 2002, 5:102-110.

32. Valko M, Leibfritz D, Moncol J, Cronin MTD, Mazur M, Telser J: Free radicals and antioxidants in normal physiological functions and human disease. Int J Biochem Cell Biol 2007, 39:44-84.

33. Van Raamsdonk JM, Hekimi S: Reactive oxygen species and aging in Caenorhabditis elegans: causal or casual relationship? Antioxid Redox Signal 2010, 13:1911-1953.

34. Van Raamsdonk JM, Hekimi S: Superoxide dismutase is dispensable for normal animal lifespan. Proc Natl Acad Sci U S A 2012, 109:5785-5790

35. Doonan R, McElwee JJ, Matthijssens F, Walker GA, Houthoofd K, Back P, Matscheski A, Vanfleteren JR, Gems D: Against the oxidative damage theory of aging: superoxide dismutases protect against oxidative stress but have little or no effect on life span in Caenorhabditis elegans. Genes Dev 2008, 22:3236-3241.

36. Burmeister C, Luersen K, Heinick A, Hussein A, Domagalski M, Walter RD, Liebau E: Oxidative stress in Caenorhabditis elegans: protective effects of the Omega class glutathione transferase (GSTO-1). FASEB J 2008, 22:343-354

37. Yen K, Patel HB, Lublin AL, Mobbs CV: SOD isoforms play no role in lifespan in ad lib or dietary restricted conditions, but mutational inactivation of SOD-1 reduces life extension by cold. Mech Ageing Dev 2009, 130:173-178

38. Van Raamsdonk JM, Hekimi S: Deletion of the mitochondrial superoxide dismutase sod-2 extends lifespan in Caenorhabditis elegans. PloS Genet 2009, 5:e1000361.

39. Pun PB, Gruber J, Tang SY, Schaffer S, Ong RL, Fong S, Ng LF, Cheah I, Halliwell B: Ageing in nematodes: do antioxidants extend lifespan in Caenorhabditis elegans? Biogerontology 2010, 11:17-30.

40. Gruber J, Ng LF, Poovathingal SK, Halliwell B: Deceptively simple but simply deceptive-Caenorhabditis elegans lifespan studies: considerations for aging and antioxidant effects. FEBS Lett 2009, 583:3377-3387.

41. Keaney M, Matthijssens F, Sharpe M, Vanfleteren J, Gems D: Superoxide dismutase mimetics elevate superoxide dismutase activity in vivo but do not retard aging in the nematode Caenorhabditis elegans. Free Radic Biol Med 2004, 37:239-250

42. Bronikowski A, Flatt T: Aging and its demographic measurement. Nat Educ Knowl 2010, 3:1-6.

43. Walker DW, McColl G, Jenkins NL, Harris J, Lithgow GJ: Evolution of lifespan in C. elegans. Nature 2000, 405:296-297.

44. Wu D, Tedesco PM, Phillips PC, Johnson TE: Fertility/longevity trade-offs under limiting-male conditions in mating populations of Caenorhabditis elegans. Exp Gerontol 2012, 47:759-763.

45. Yang $W$, Tiffany-Castiglioni E: The bipyridyl herbicide paraquat produces oxidative stress-mediated toxicity in human neuroblastoma SH-SY5Y cells: relevance to the dopaminergic pathogenesis. J Toxicol Env Heal A 2005, 68:1939-1961.

46. Bus JS, Aust SD, Gibson JE: Paraquat toxicity: proposed mechanism of action involving lipid peroxidation. Environ Health Perspect 1976, 16:139-146.

47. Joyner-Matos J, Hicks KA, Cousins D, Keller M, Denver DR, Baer CF, Estes S: Evolution of a higher intracellular oxidizing environment in Caenorhabditis elegans under relaxed selection. PLoS One 2013, 8:e65604.

48. Stiernagle T: Maintenance of $C$. elegans. In WormBook. Edited by The C. elegans Research Community. 2006. doi/10.1895/wormbook.1.101.1, http://www.wormbook.org

49. Winterbourn CC: Reconciling the chemistry and biology of reactive oxygen species. Nat Chem Biol 2008, 4:278-286.

50. Ishii N, Takahashi K, Tomita S, Keino T, Honda S, Yoshino K, Suzuki K A methyl viologen-sensitive mutant of the nematode Caenorhabditis elegans. Mutat Res 1990, 237:165-171.

51. Hassan HM, Fridovich I: Intracellular production of superoxide radical and of hydrogen peroxide by redox active compounds. Arch Biochem Biophys 1979, 196:385-395.
52. Gems D, Doonan R: Oxidative stress and ageing in the nematode Caenorhabditis elegans. In Oxidative Stress in Aging. Humana Press, New York, NY; 2008:81-110

53. Anderson JL, Albergotti L, Ellebracht B, Huey RB, Phillips PC: Does thermoregulatory behavior maximize reproductive fitness of natural isolates of Caenorhabditis elegans? BMC Evol Biol 2011, 11:157.

54. Anderson JL, Albergotti L, Proulx S, Peden C, Huey RB, Phillips PC: Thermal preference of Caenorhabditis elegans: a null model and empirical tests. J Exp Biol 2007, 210(Pt 17):3107-3116.

55. Zuo W, Moses ME, West GB, Hou C, Brown JH: A general model for effects of temperature on ectotherm ontogenetic growth and development. Proc Biol Sci 2011, 279:1840-1846.

56. Harvey SC, Viney ME: Thermal variation reveals natural variation between isolates of Caenorhabditis elegans. J Exp Zool B Mol Dev Evol 2007, 308:409-416.

57. Byerly $L$, Cassada RC, Russell RL: The life cycle of the nematode Caenorhabditis elegans, I: wild-type growth and reproduction. Dev Biol 1976, 51:23-33.

58. Goranson N, Ebersole J, Brault S: Resolving an adaptive conundrum: reproduction in Caenorhabditis elegans is not sperm-limited when food is scarce. Evol Ecol Res 2005, 7:325-333.

59. Hubbard EJA, Greenstein D, The C. elegans Research Community: Introduction to the Germ line. In WormBook, WormBook 2005. doi:10.1895/ wormbook.1.18.1, http://www.wormbook.org

60. Cutter AD: Sperm-limited fecundity in nematodes: how many sperm are enough? Evolution 2004, 58:651-655.

61. Garigan D, Hsu A-L, Fraser AG, Kamath RS, Ahringer J, Kenyon C: Genetic analysis of tissue aging in Caenorhabditis elegans: a role for heat-shock factor and bacterial proliferation. Genetics 2002, 161:1101-1112.

62. Gems D, Riddle DL: Genetic, behavioral and environmental determinants of male longevity in Caenorhabditis elegans. Genetics 2000, 154:1597-1610.

63. Sutphin GL, Kaeberlein M: Measuring Caenorhabditis elegans life span on solid media. J Vis Exp 2009, 27:1152.

64. Walker G, Houthoofd K, Vanfleteren JR, Gems D: Dietary restriction in C elegans: from rate-of-living effects to nutrient sensing pathways. Mech Ageing Dev 2005, 126:929-937.

65. Hicks KA, Howe DK, Leung A, Denver DR, Estes S: In vivo quantification reveals extensive natural variation in mitochondrial form and function in Caenorhabditis briggsae. PLoS One 2012, 7:e43837.

66. Hicks KA, Denver DR, Estes S: Natural variation in Caenorhabditis briggsae mitochondrial form and function suggests a novel model of organelle dynamics. Mitochondrion 2013, 13:44-51.

67. Zielonka J, Kalyanaraman B: Hydroethidine- and MitoSOX-derived red fluorescence is not a reliable indicator of intracellular superoxide formation: another inconvenient truth. Free Radic Biol Med 2010, 48:983-1001.

68. Estes S, Phillips PC, Denver DR, Thomas WK, Lynch M: Mutation accumulation in populations of varying size: the distribution of mutational effects for fitness correlates in Caenorhabditis elegans. Genetics 2004, 166:1269-1279.

69. Keightley PD, Davies EK, Peters AD, Shaw RG: Properties of ethylmethane sulfonate-induced mutations affecting life-history traits in Caenorhabditis elegans and inferences about bivariate distributions of mutation effects. Genetics 2000, 156:143-154.

70. Pletcher SD, Neuhauser C: Biological aging — criteria for modeling and a new mechanistic model. Int J Mod Phys C 2000, 11:525-546.

71. Bronikowski AM, Morgan TJ, Garland T, Carter PA: The evolution of aging and age-related physical decline in mice selectively bred for high voluntary exercise. Evolution (N Y) 2006, 60:1494-1508.

72. Philips PC, Arnold SJ: Hierarchical comparison of genetic variance-covariance matrices, l: using the Flury hierarchy. Evolution (N Y) 1999, 53:1506-1515.

73. Altun ZF, Hall DH: Introduction to C. elegans anatomy. In WormAtlas. 2009. doi:10.3908/wormatlas.1.1.

74. Gems D, Riddle DL: Defining wild-type life span in Caenorhabditis elegans. J Gerontol A Biol Sci Med Sci 2000, 55:B215-B219.

75. Tawe WN, Eschbach ML, Walter RD, Henkle-Duhrsen K: Identification of stress-responsive genes in Caenorhabditis elegans using RT-PCR differential display. Nucleic Acids Res 1998, 26:1621-1627.

76. Braeckman BP, Houthoofd K, Vanfleteren JR, The C. elegans Research Community: Intermediary Metabolism. In WormBook. WormBook 2009; doi:10.1895/wormbook.1.101.1, http://www.wormbook.org

77. Hartman P, Childress E, Beyer T: Nematode development is inhibited by methyl viologen and high oxygen concentrations at a rate inversely proportional to life span. J Gerontol A Biol Sci Med Sci 1995, 50:B322-B326. 
78. Jonassen T, Marbois BN, Faull KF, Clarke CF, Larsen PL: Development and fertility in Caenorhabditis elegans clk-1 mutants depend upon transport of dietary coenzyme Q8 to mitochondria. J Biol Chem 2002, 277:45020-45027.

79. Goerlich O, Quillardet P, Hofnung M: Induction of the SOS response by hydrogen peroxide in various Escherichia coli mutants with altered protection against oxidative DNA damage. J Bacterio/ 1989, 171:6141-6147.

80. Blanchard JL, Wholey W-Y, Conlon EM, Pomposiello PJ: Rapid changes in gene expression dynamics in response to superoxide reveal SoxRS-dependent and independent transcriptional networks. PLoS One 2007, 2:e1186.

81. Greenberg JT, Monach P, Chou JH, Josephy PD, Demple B: Positive control of a global antioxidant defense regulon activated by superoxide-generating agents in Escherichia coli. Proc Natl Acad Sci U S A 1990, 87:6181-6185.

82. Davison CL, Papirmeister B: Bacteriostasis of Escherichia coli by the Herbicide Paraquat. Exp Biol Med 1971, 136:359-364

83. Hodgkin J, Barnes TM: More is not better: brood size and population growth in a self-fertilizing nematode. Proc Biol Sci 1991, 246:19-24.

84. McElwee J, Bubb K, Thomas JH: Transcriptional outputs of the Caenorhabditis elegans forkhead protein DAF-16. Aging Cell 2003, 2:111-121.

85. Henderson ST, Johnson TE: daf-16 integrates developmental and environmental inputs to mediate aging in the nematode Caenorhabditis elegans. Curr Biol 2001, 11:1975-1980.

86. Speakman JR, Selman C: The free-radical damage theory: accumulating evidence against a simple link of oxidative stress to ageing and lifespan. Bioessays 2011, 33:255-259.

doi:10.1186/s12862-014-0161-8

Cite this article as: Smith et al:: Endogenous ROS levels in C. elegans under exogenous stress support revision of oxidative stress theory of life-history tradeoffs. BMC Evolutionary Biology 2014 14:161.

\section{Submit your next manuscript to BioMed Central and take full advantage of:}

- Convenient online submission

- Thorough peer review

- No space constraints or color figure charges

- Immediate publication on acceptance

- Inclusion in PubMed, CAS, Scopus and Google Scholar

- Research which is freely available for redistribution 